\section{FRI0333 B-CELLS DEPLETION AS RESCUE THERAPY FOR EXTRA-CRITERIA MANIFESTATIONS OF PRIMARY ANTIPHOSPHOLIPID SYNDROME}

S. Sciascia ${ }^{1}$, M. Radin ${ }^{1}$, I. Cecchi ${ }^{1}$, E. Rubini ${ }^{1}$, D. Rossi ${ }^{2}$, M. Bazzan $^{3}$, D. Roccatello ${ }^{1}{ }^{1}$ Department of Clinical and Biological Sciences, Università Degli Studi Di Torino; ${ }^{2}$ Ospedale S. Giovanni Bosco, Turin, Italy; ${ }^{3}$ UOSD Hematology and Thrombosis Unit, Ospedale S. Giovanni Bosco, Turin, Italy

Background: While immunosuppressive drugs may be helpful in patients with active systemic autoimmune diseases, their use in antiphospholipid syndrome (APS) is still controversial, and mainly limited to very selected cases of catastrophic APS or in severe cases refractory to standard therapy. ${ }^{1}$ B-cells are likely to play a central role in the generation of the aPL-induced clinical manifestations of the disease, so they might constitute a logical therapeutic target in APS.

Objectives: To investigate PAPS patients with extra-criteria manifestations of APS treated with Rituximab (RTX) as a rescue therapy.

Methods: We retrospectively retrieved data from patients who attended the $S$. Giovanni Bosco Hospital, Turin, Italy, who met the following inclusion criteria: a) persistent aPL positivity and fulfilled the Sydney criteria for PAPS $\left[{ }^{2}\right.$ b) presented with at least 1 extra-criteria manifestation; c) were treated with RTX as a rescue therapy (e.g. because they were refractory/intolerant/contraindicated to standard therapy) for the management of extra-criteria manifestations of APS.

Results: This retrospective study included 7 consecutive PAPS patients [median age 53 (range 38-66), female 6:1]. Table 1 resumes the characteristics of the PAPS patients included in the study. Six patients presented with severe thrombocytopenia (plts $<50.000 / \mathrm{mm}^{3}$ ) and 1 patient presented with recurrent superficial venous thrombosis ( 3 events in 6 months despite ongoing anticoagulation therapy with VKA). Previous therapies included intravenous immunoglobulins (5 patients) and high doses of steroids ( 3 patients). One patient received RTX as rescue therapy as a steroid-sparing agent because of the high cardiovascular risk (high body mass index, uncontrolled arterial hypertension, and diabetes). We observed a full response after treatment with RTX in 6 out of 7 patients $(86 \%, 5$ with thrombocytopenia and 1 with recurrent superficial thrombosis). One patient who did not respond to the B-cell depletion therapy, and was treated with a splenectomy 1 month after RTX therapy and platelets levels normalised 3 months after the procedure. Overall, median time free from relapse was 27.5 months (range 4-97), no adverse events were reported, no patients developed infections.

Abstract FRI0333 - Table 1. Patients with PAPS with extra-criteria manifestations that followed a B-cells depleting protocol from the experience of our centre

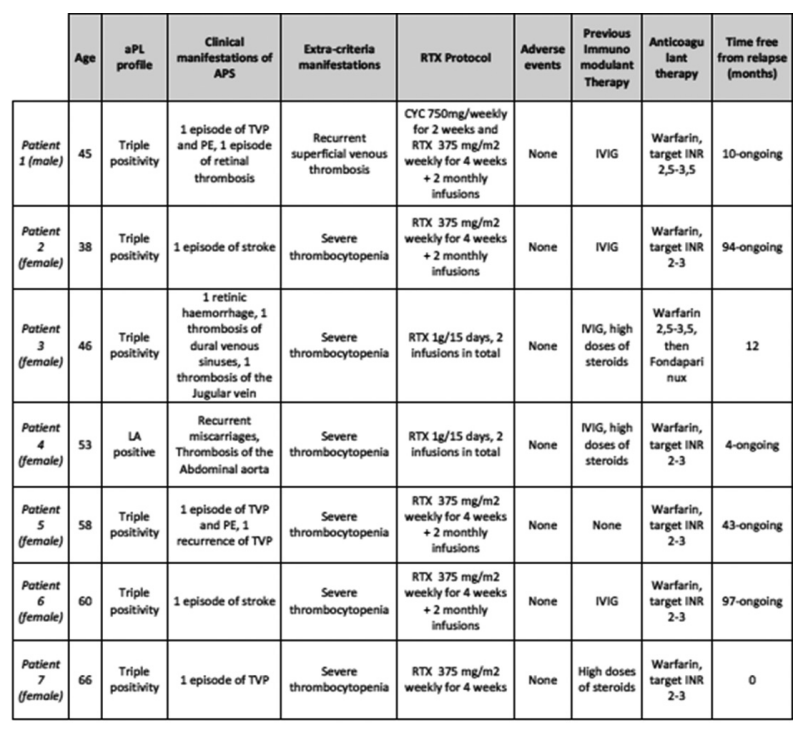

APS Antiphospholipid Syndrome, UC undifferentiated connective tissue disease, CYC cyclophosphamide, RTX rituximab, AZA

azathioprine, MTX methotrexate, OVT deep vein thrombosis, PE pulmonary embolism

Conclusions: In selected case of patients with PAPS, RTX can represent a safe and efficacious therapeutic tool to manage the extra-criteria manifestations of the syndrome, especially when standard approaches have failed or cannot be pursued.

\section{REFERENCES:}

[1] Erre GL, Pardini S, Faedda R, Passiu G. Effect of rituximab on clinical and laboratory features of antiphospholipid syndrome: a case report and a review of literature. Lupus 2008:17:50-5. doi:10.1177/0961203307085251

[2] Miyakis S, Lockshin MD, Atsumi T, Branch DW, Brey RL, Cervera R, et al. International consensus statement on an update of the classification criteria for definite antiphospholipid syndrome (APS). J Thromb Haemost 2006;4:295-306. doi:10.1111/j.1538-7836.2006.01753.x

Disclosure of Interest: None declared

DOI: 10.1136/annrheumdis-2018-eular.2157

\section{FRI0334 COMPARISON OF DIFFERENT DEFINITIONS OF REMISSION IN SYSTEMIC LUPUS ERYTHEMATOSUS (SLE) - A STUDY BASED ON THE BLISS-76 CLINICAL TRIAL}

S. Emamikia ${ }^{1}$, C. Gentline ${ }^{1}$, E. Arkema ${ }^{2}$, L. Arnaud ${ }^{3}$, K. Chatzidionysiou ${ }^{1}$, R. van Vollenhoven ${ }^{1,4}$. ${ }^{1}$ Department of Medicine, ${ }^{2}$ Clinical Epidemiology Unit, Karolinska Institute, Stockholm, Sweden; ${ }^{3}$ Department of Rheumatology, Université de Strasbourg, Strasbourg, France, ${ }^{4}$ Amsterdam Rheumatology and Immunology Center, Amsterdam, Netherlands

Background: Standardisation of a definition of disease remission in SLE is one of the most important unmet needs in rheumatology and up to today there is no gold standard for the definition of remission. ${ }^{1}$

Objectives: An international task force consisting of 60 rheumatologists, nephrologists, dermatologists, clinical immunologists and patient representatives agreed on various definitions of remission in SLE (DORIS). ${ }^{2}$ Our aim was to apply these definitions on a clinical trial.

Methods: This is a post-hoc analysis of a prospective randomised controlled tria (RCT) in SLE, the BLISS-76 clinical trial. ${ }^{3}$ We have applied two DORIS definitions (table 1) at three time points, week (wk) 24, 52 and 76. The patient could be in remission on or off treatment. Remission on treatment allowed maintenance antimalarials, low dose glucocorticoids (GCs) (prednisone $\leq 5 \mathrm{mg} /$ day or equivalent), maintenance immunosuppressives and maintenance biologics. Remission off treatment allowed maintenance anti-malarials only. Additionally, we applied each definition where the remission on treatment allowed a GC dose $\leq 10 \mathrm{mg} /$ day (not a part of the original DORIS-definitions).

Abstract FRI0334 - Table 1. DORIS definitions of remission in SLE

\begin{tabular}{|c|c|c|c|c|}
\hline Definition & $1 a$ & $1 \mathrm{~b}$ & $3 a$ & 3b \\
\hline CSLEDAI $=0$ & $\mathrm{v}$ & $\mathrm{v}$ & - & - \\
\hline$P h G A<0.5$ & $v$ & $v$ & $v$ & $v$ \\
\hline Serology & Regardless & Normal & Regardless & Normal \\
\hline BILAG D/E only & - & - & $v$ & $v$ \\
\hline \multicolumn{5}{|c|}{$\begin{array}{l}\text { cSLEDAI: clinical Systemic Lupus Erythematosus Diseasc Activity Index; } \\
\text { PLGA Physician Global Assessment; } \\
\text { BILAG: British Isles Lupus Assessment Group } \\
\text { Note: Anti-double stranded DNA and complement (C3 and C4) is included in serology. These definitions were } \\
\text { first tested (a) regardless of serology and then (b) normal serology (negative anti-dsDNA antibodies AND } \\
\text { normal complement). }\end{array}$} \\
\hline
\end{tabular}

Abstract FRI0334 - Table 2. Percent of Patients who started the BLISS-76 trial $(n=819)$ who were in remission at week 24 , week 52 , and week 76 using different definitions

\begin{tabular}{|c|c|c|c|c|c|c|}
\hline & $\begin{array}{l}\text { Definition la, } \\
\text { On treatment } \\
\text { (GCss } \mathrm{mg} \\
\text { allowed) }\end{array}$ & \begin{tabular}{|l|} 
Definition \\
$1 \mathrm{a}$, On \\
treatment \\
(GCs $\leq 10 \mathrm{mg}$ \\
allowed) \\
\end{tabular} & \begin{tabular}{|l|} 
Definition 1a, \\
Off treatment
\end{tabular} & \begin{tabular}{|l|} 
Definition Ib, \\
On treatment \\
(GCs $\leq 5 \mathrm{mg}$ \\
allowed)
\end{tabular} & \begin{tabular}{|l|} 
Definition Ib, On \\
treatment \\
(GCs $\leq 10 \mathrm{mg}$ \\
allowed)
\end{tabular} & \begin{tabular}{|l} 
Definition Ib, \\
Off treatment
\end{tabular} \\
\hline $\begin{array}{l}\%(\mathrm{n}) \text { in } \\
\text { remission W24 }\end{array}$ & $\begin{array}{l}.2 \\
(34)\end{array}$ & $\begin{array}{l}77.3 \\
(60)\end{array}$ & $\begin{array}{l}0.4 \\
\text { (3) }\end{array}$ & $\begin{array}{l}1.0 \\
(8)\end{array}$ & $\begin{array}{l}1.7 \\
\text { (14) }\end{array}$ & $\begin{array}{l}0.1 \\
\text { (1) }\end{array}$ \\
\hline $\begin{array}{l}\% \text { (n) in } \\
\text { remission W52 }\end{array}$ & $\begin{array}{l}7.4 \\
\text { (61) }\end{array}$ & $\begin{array}{l}11.8 \\
(97)\end{array}$ & $\begin{array}{l}0.7 \\
\text { (6) }\end{array}$ & $\begin{array}{l}2.6 \\
\text { (21) }\end{array}$ & $\begin{array}{l}3.2 \\
(26)\end{array}$ & $\begin{array}{l}0.5 \\
\text { (4) }\end{array}$ \\
\hline \multirow[t]{2}{*}{$\begin{array}{l}\%(\mathrm{n}) \text { in } \\
\text { remission W76 }\end{array}$} & $\begin{array}{l}9.5 \\
(78)\end{array}$ & $\begin{array}{l}13.8 \\
\text { (113) }\end{array}$ & $\begin{array}{l}1.0 \\
(8)\end{array}$ & $\begin{array}{l}3.2 \\
(26)\end{array}$ & $\begin{array}{l}4.4 \\
(36)\end{array}$ & $\begin{array}{l}0.4 \\
\text { (3) }\end{array}$ \\
\hline & \begin{tabular}{|l} 
Definition 3a, \\
On treatment \\
(GCS $\leq 5 \mathrm{mg}$ \\
allowed)
\end{tabular} & \begin{tabular}{|l|} 
Definition \\
3a, on \\
treatment \\
(GCS s $10 \mathrm{mg}$ \\
allowed
\end{tabular} & \begin{tabular}{|l|} 
Definition 3a, \\
Off treatment
\end{tabular} & \begin{tabular}{|l} 
Definition 3b, \\
On treatment \\
(GCs $\leq 5 \mathrm{mg}$ \\
allowed)
\end{tabular} & \begin{tabular}{|l|} 
Definition $3 \mathrm{~b}$, On \\
treatment (GCs \\
$\leq 10 \mathrm{mg}$ allowed) \\
\end{tabular} & $\begin{array}{l}\text { Definition 3b, } \\
\text { Off treatment }\end{array}$ \\
\hline $\begin{array}{l}\% \text { (n) in } \\
\text { renission W24 }\end{array}$ & $\begin{array}{l}0.9 \\
\text { (7) }\end{array}$ & $\begin{array}{l}2.2 \\
(18)\end{array}$ & 0 & $\begin{array}{l}0.5 \\
\text { (4) }\end{array}$ & $\begin{array}{l}0.6 \\
\text { (5) }\end{array}$ & 0 \\
\hline $\begin{array}{l}\% \text { (n) in } \\
\text { remission W52 }\end{array}$ & $\begin{array}{l}1.3 \\
\text { (11) }\end{array}$ & $\begin{array}{l}2.8 \\
(23)\end{array}$ & $\begin{array}{l}0.1 \\
\text { (1) }\end{array}$ & $\begin{array}{l}0.7 \\
(6)\end{array}$ & $\begin{array}{l}1.1 \\
(9)\end{array}$ & $\begin{array}{l}0.1 \\
\text { (1) }\end{array}$ \\
\hline $\begin{array}{l}\% \text { (n) in } \\
\text { remission W76 }\end{array}$ & $\begin{array}{l}2.9 \\
(24)\end{array}$ & $\begin{array}{l}4.5 \\
(37)\end{array}$ & $\begin{array}{l}0.1 \\
\text { (1) }\end{array}$ & $\begin{array}{l}1.6 \\
(13)\end{array}$ & $\begin{array}{l}2.3 \\
(19)\end{array}$ & $\begin{array}{l}0.1 \\
\text { (1) }\end{array}$ \\
\hline
\end{tabular}

Def1a:cSLEDAl=0, PhGA $<0.5$ and normal serology

Def $1 \mathrm{~b}$ : cSLEDAl=0, PhGA $<0.5$ and regardless of serology

Def3a:BILAG D/E only, PhGA $<0.5$ and normal serology

Def $3 b$ : BILAG D/E only, PhGA $<0.5$ and regardless of serology

GCs: Glucocorticoids

On treatment: maintenance anti-malarials, low dose glucocorticoids (prednisone $\leq 5 \mathrm{mg} /$ day or equivalent), maintenance immunosuppressives, and maintenance biologics are allowed. Off treatment: maintenance anti-malarials only is allowed.

Results: There were 819 patients enrolled in BLISS-76. The proportions of patients that fulfilled remission according to the above definitions are shown in table 2 . The highest point prevalence $(9.5 \%)$ was when definition 1a on treatment was applied at wk 76. As expected, even more patients fulfilled definition 1a when a GC dose $\leq 10 \mathrm{mg} /$ day was allowed at wk 76 (13.8\%). More patients fulfilled the 